\title{
Ciencia y tecnología, perspectiva para Colombia
}

\section{Laura Mendivil Anaya*}

\section{Resumen}

El presente articulo hace un recorrido por los siguientes temas: la importancia de la inversión en Ciencia y Tecnología, el panorama mundial de la inversión en Ciencia y Tecnología, asi como la Ciencia y la Tecnología en Colombia, profundizando en el Sistema de Ciencia y Tecnología, el presupuesto asignado y las posibles rutas para cualificar el tema en el país.

Palabras Clave: Ciencia y Tecnología, Investigación y Desarrollo, presupuesto Ciencia y Tecnología, Colombia.

\section{Abstract}

This article makes an overview of the following subjects: the importance of investing in science and technology, the world-wide panorama of science and technology and science and technology in Colombia, emphasizing the Science and Technology System, the budget assigned to this area, and the possible ways to improve this topic in Colombia.

El conocimiento, la educación y los desarrollos tecnológicos dan cuenta de los procesos de un conjunto de recursos humanos, técnicos, el acceso a la información y sobre todo, las políticas que un Estado implementa para organizar tales recursos. En un mundo cada vez más globalizado, la brecha en la capacidades científicas y tecnológicas entre los países es una de las más fehacientes manifestaciones del subdesarrollo y el desarrollo según la importancia $y$ adelanto que se le de a las mismas.
Es por ello que la inversión en ciencia y tecnología y la eficiencia en dicha inversión es esencial para que una sociedad responda al entendimiento de su entorno y sobre todo, se fundamente el desarrollo y dinamice el cambio social. Concientes de esa realidad, países como EE.UU. han formulado programas muy ambiciosos a 20 años que abastecerán de recursos tanto al cálculo como a la experimentación de súper calculadoras, súper redes inteligentes, biotecnología, nano- tecnologías, robótica, entre otros, con un esquema de trabajo mancomunado entre científicos y la industria, quienes se encargarían de realizar y poner en marcha los productos y el saber que se genera. Así, se genera "un perfecto ensamble entre la investigación científica y tecnológica y la actividad productiva, que retroalimenta el sistema hasta niveles insospechados"

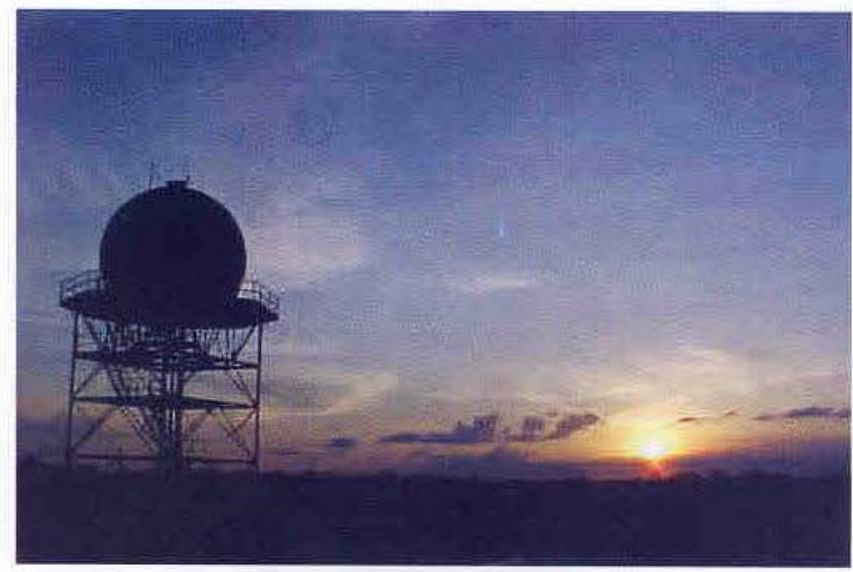

* Politóloga, Especialista en Gobierna y Politicas Püblicas. Asesora de investigación IMA

'Leguizamón, Eduardo Sixto. Ciencia y Tecnologia, la Argentina y el Mundo. Disponible en: http://www.fcagr.unreduar/lnvestigacion/revista/rev5/v.htm 
Con relación a la inversión mundial en Investigación y Desarrollo, según datos del año 2000, únicamente EE.UU. y Canadá aportan el $42,5 \%$ de la inversión mundial, mientras la Unión Europea aporta el 24\%, seguidos por Japón con $15.6 \%$ y el resto de Asia con $10.1 \%$. En ese panorama América Latina ocupa un rezagado 6 puesto con $\tan$ solo $1.6 \%$.

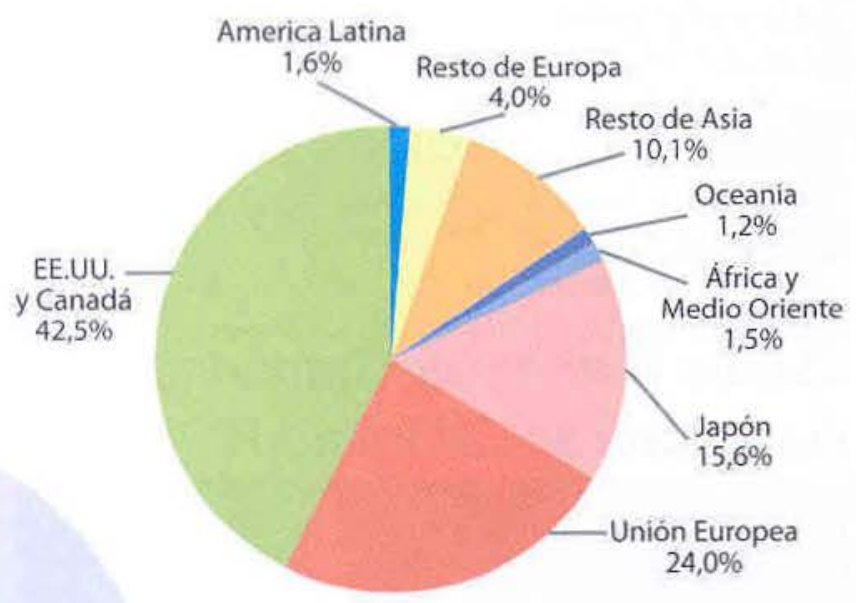

\section{Gráfico de Inversión Mundial en I+D 2000 \\ Fuente: OCDE, UNESCO, RICYT}

En ese escenario ¿Cómo se encuentra Colombia? El país es uno de los que menos presupuesto estatal destina para el desarrollo de la ciencia y la tecnología, tan sólo el $1.5 \%$ del PIB. Según un estudio de The Global Technology Revolutions 2021, de la Rand National Security Research Division de EE.UU., Colombia es ubicada en el grupo de los "paises en desarrollo científico", junto con Brasil, Chile, México, Turquía, Sur África e Indonesia. Ésa es la tercera categoría de cuatro, en la que la última son los "paises científicamente rezagados", la segunda "científicamente competentes" y la primera, los "científicamente avanzados". Tal ubicación de Colombia correspondería por un lado, a la volatilidad de la asignación de recursos, y por el otro, a que los recursos asignados a la investigación y desarrollo son empleados simultáneamente para la capacitación y formación de investigadores, como a la investigación en si misma, lo cual disminuye el potencial de la investigación.

A su vez, factores como la pobreza, la precariedad en los servicios públicos, la inestabilidad política y los pocos recursos económicos que caracteriza a la mayoría de los paises latinoamericanos, son barreras para los logros en el uso de la ciencia y tecnología descritos por la Rand, entre los que se encuentran las áreas de biotecnología, nanotecnología, tecnologías de materiales y de la información. Para poder determinar tales barreras, el estudio calculó en términos de porcentajes tanto las barreras a los usos, como las fuerzas impulsoras a los mismos, en dónde los países "cientificamente avanzados" como Alemania y Canadá, tienen un $30 \%$ de barreras y cuentan con el $100 \%$ de fuerzas impulsoras; por su parte, Estados Unidos tiene $40 \%$ de barreras y $100 \%$ de fuerzas. También Australia, Japón y Corea, con el $30 \%$ y $90 \%$. Finalmente, Israel, que tiene el $40 \%$ de barreras y el $90 \%$ de fuerzas impulsoras. Un caso especial en esta perspectiva es China, que a pesar de tener un $70 \%$ de barreras, cuenta con más del $50 \%$ de oportunidades. El estudio evidenció que las barreras en Colombia están en un $70 \%$ mientras que las fuerzas impulsoras en un $10 \%$.

Pero cabe preguntarse el por qué de esa situación. Para ello, se hará un pequeño recorrido por la situación de la Ciencia y Tecnologia en Colombia, para culminar con unas posibles soluciones para cualificar el tema en el pais.

Fue la ley 29 de 1990 la que institucionalizó el Sistema Nacional de Ciencia y Tecnología, como resultado de la Misión de Ciencia y Tecnología, Misión de Sabios convocada por el gobierno Barco a finales de la década de los 80. De esta forma, los organismos de dirección y coordinación del Sistema son el Consejo Nacional de Ciencia y Tecnología, los Consejos de Programas Nacionales, las Comisiones Regionales, los Consejos de Programas Regionales y el Comité de Formación de Recursos Humanos para la Ciencia y la Tecnología. Sin embargo, éstos no constituyen estructuras administrativas independientes ni planta de personal propia.

En este sistema el organismo central y preponderante es COLCIENCIAS, el cual ejerce las veces de secretaria técnica, éste se apoya en seis áreas : Investigación Fundamental en Ciencias Básicas, Sociales y Humanas o Gestión del Conocimiento, de las Aplicaciones Sociales y de la Convergencia Tecnológica o De la Materia y la Energía o Procesos Biológicos, Agroalimentarios y Biodiversidad o El Ser Humano y su Entorno o Educación, la Cultura y las Instituciones ${ }^{2}$. COLCIENCIAS, en su presupuesto de inversión acumuló entre 2001 y 2004 una inversión de $\$ 299.877 .3$ millones, y éste fue para el 2005 de $\$ 87.266$ millones, aumentando por encima del $16 \%$ con relación a la media cuatrienio del año anterior. 


\section{Tabla de ejecución presupuestal año 2005 COLCIENCIAS}

\begin{tabular}{|c|c|c|}
\hline $\begin{array}{l}\text { ESTRATEGIAS } \\
\text { INSTITUCIONALES }\end{array}$ & EJECUCIÓN & $\%$ \\
\hline $\begin{array}{l}\text { PROMOCIÓN DE LA } \\
\text { INVESTIGACIONN }\end{array}$ & 30.029 .052 .615 & 34,41 \\
\hline $\begin{array}{l}\text { FORTALECIMIENTO DE LA } \\
\text { CAPACIDAD INSTITUCIONAL }\end{array}$ & 4.916 .446 .150 & 5,63 \\
\hline $\begin{array}{l}\text { ESTIMUULO A LA INNOVACIÓN } \\
\text { Y AL DESARROLLO } \\
\text { TECNOLOGICO }\end{array}$ & 27.425 .131 .555 & 31,43 \\
\hline $\begin{array}{l}\text { CAPACITACION EN } \\
\text { INVESTIGACIONY } \\
\text { DESARROLLO EN AREAS } \\
\text { ESTRATEEGICAS }\end{array}$ & $21.728 .753 .829 t$ & 24,9 \\
\hline OTRAS ESTRATEEGICAS & 3.166 .653 .872 & 3,63 \\
\hline TOTAL & 87.266 .038 .021 & 100 \\
\hline
\end{tabular}

\section{Tabla de ejecución presupuestal año 2006 COLCIENCIAS}

\begin{tabular}{|c|c|c|}
\hline $\begin{array}{l}\text { ESTRATEGIAS } \\
\text { INSTITUCIONALES }\end{array}$ & EJECUCIÓN & $\%$ \\
\hline $\begin{array}{l}\text { PROMOCIÓNDE LA } \\
\text { INVESTIGACIOON }\end{array}$ & 50.983 .784 .120 & $41,36 \%$ \\
\hline $\begin{array}{l}\text { FORTALECIMIENTO DE LA } \\
\text { CAPACIDAD INSTITUCIONAL }\end{array}$ & 7.600 .433 .677 & $6,17 \%$ \\
\hline $\begin{array}{l}\text { ESTIMULO A LA INNOVACIÓNY } \\
\text { AL DESARROLLO TECNOLOG. }\end{array}$ & 36.294 .999 .999 & $29,45 \%$ \\
\hline $\begin{array}{l}\text { CAPACITACIÓN EN } \\
\text { INVESTIGACIOONY } \\
\text { DESARROLLO EN AREAS } \\
\text { ESTRATEGICAS }\end{array}$ & 22.796 .250 .000 & $18,50 \%$ \\
\hline $\begin{array}{l}\text { FORTALECIMIENTO DE LA } \\
\text { CAPACIDAD REGIONAL EN CYT }\end{array}$ & 3.300 .000 .000 & $2,68 \%$ \\
\hline $\begin{array}{l}\text { APROPIACION SOCIAL DELA } \\
\text { CIENCIA Y LA TECNOLOGIA }\end{array}$ & 1.819 .800 .000 & $1,48 \%$ \\
\hline $\begin{array}{l}\text { TOTAL PRESUPUESTO DE } \\
\text { INVERSIÓN COLCIENCIAS }\end{array}$ & 122.795 .267 .796 & $99,63 \%$ \\
\hline Distribución porcentual & $99,6 \%$ & \\
\hline
\end{tabular}

Otro indicador relevante a la hora de medir los resultados con respecto a la Ciencia y Tecnología es la formación doctoral como una estrategia para fortalecer la investigación, en dónde según el Observatorio para la Ciencia y la Tecnología, por un lado, ha habido un gran avance desde 1994 cuando se graduó el primer doctor en el pais, hasta 2005 cuando se contaban más de 160 doctores. Así mismo, se esperaba que para el 2006, se graduaran muchos más, gracias a las becas otorgadas por COLCIENCIAS que superaban las 300 . Sin embargo, el aumento es lento, ya que para igualar el ritmo de los paises más desarrollados, se requeriría el grado de 5000 doctores por año.

\section{Cuadro Número de Graduandos de Doctorado en Colombia 1994-2002}

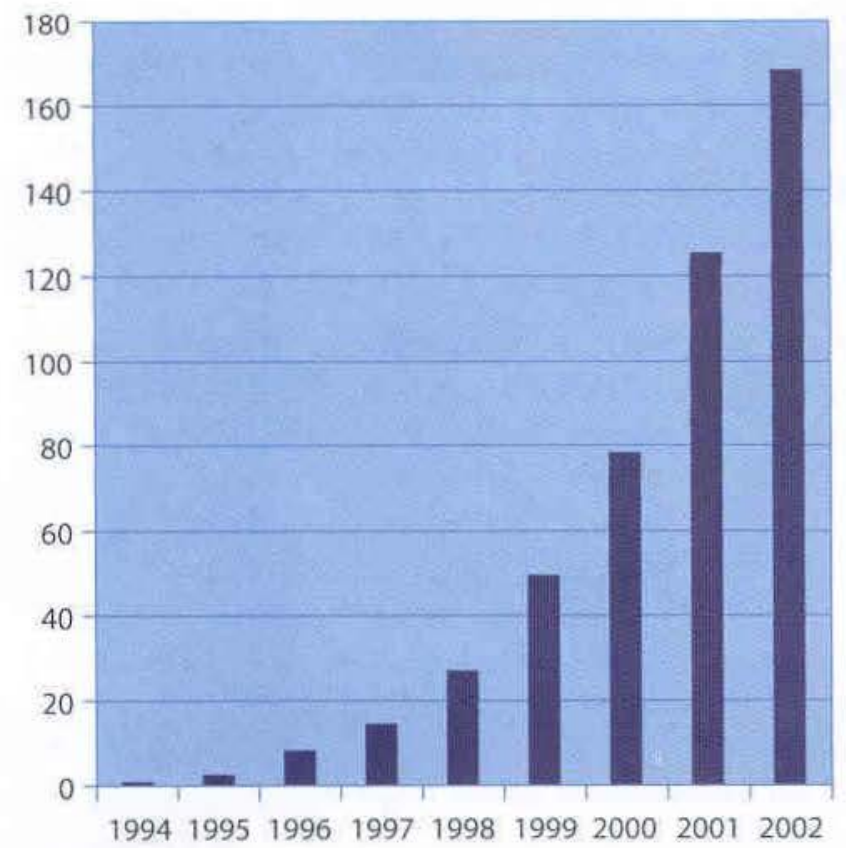

Fuente: Observatorio de Ciencias y Tecnología

Ante la realidad descrita, ¿Qué alternativas tiene el país? Colombia ha hecho grandes esfuerzos por reducir las diferencias existentes en el campo de la Ciencia y la Tecnología con los paises vecinos. Una posible solución es hacer esfuerzos mancomunados desde el Estado, las universidades, los centros de investigación y la industria. Así mismo, es importante determinar algún mecanismo de medición que permita establecer hasta qué punto la inversión es para la capacitación de investigadores y hasta qué punto es para Investigación y Desarrollo. 
El país debe seguir fortaleciendo y aumentando sus programas de doctorado, lo que a su vez probablemente coadyuvará a la generación de más grupos de investigación, con una mejor calidad. Otra de las propuestas que se han hecho es la de crear un Ministerio de Ciencia y Tecnología; lo anterior puede ser posible con la transformación del actual COLCIENCIAS en un ministerio, para de esta forma, evitar el riesgo de la burocratización.

Finalmente, si el Estado colombiano no decide apostarle a la Ciencia y Tecnología como una de sus prioridades, el pais seguirá estando rezagado y a la sombra del desarrollo mundial, muy por debajo de los estándares de paises como Brasil, México, Chile, Venezuela y Cuba.

\section{BIBLIOGRAFIA}

Castro Luis Fernando. En ciencia y tecnologia DESALENTADORFUTURO PARA COLOMBIA. Disponible en: http://aupec.univalle.edu.co/informes/feb99/ investigaciones.html

Cepeda Paulo. Inversiones en ciencia y tecnologia son insuficientes para competir en el TLC: Conciencias. Disponible en:

http://www.portafolio.com.co/proy_porta_online/ tlc/opi_tIC/ARTICULO-WEB NOTA_INTERIOR_PORTA1785693.html

COLCIENCIAS. Presentación. Disponible en: http:// zulia.colciencias.gov.co:8098/portalcol/

Departamento Nacional de Planeación. Documento CONPES 3080. Política Nacional de Ciencia y Tecnología. 2000-2002. Bogotá. 2000.

Leguizamón, Eduardo Sixto. Ciencia y Tecnología, la Argentina y el Mundo. Disponible en: http://www. fcagr.unr.edu.ar/Investigacion/revista/rev5/v.htm

Observatorio Colombiano de Ciencia y Tecnología. Indicadores de Ciencia y Tecnología, Colombia 2005. Primera Edición. Bogotá. 2005

Rodriguez Gabriela. ¿Ministerio de Ciencia y Tecnología?. Disponible en: http://unperiodico.unal. edu.co/ediciones/104/13a.html

Colombia, por fuera del mundial 2020 en ciencia y tecnologia. Disponible en: http:// unperiodico.unal.edu.co/ediciones/104/13.html

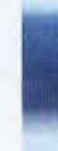

